\title{
Methotrexate in Pediatric Osteosarcoma: Response and Toxicity in Relation to Genetic Polymorphisms and Dihydrofolate Reductase and Reduced Folate Carrier 1 Expression
}

Ana Patiño-García, PhD, Marta Zalacaín, PhD, Lucía Marrodán, BSc, Mikel San-Julián, Md, PhD, and Luis Sierrasesúmaga, Md, PhD

From the Laboratory of Pediatrics, University of Navarra/University Clinic (A.P., M.Z., L.M., L.S.), and the Department of Traumatology and Orthopaedic Surgery, University Clinic of Navarra (M.S.-J.), Pamplona, Spain.

ABSTRACT

Reprint requests: Ana Patiño-García, $\mathrm{PhD}$

Laboratory of Pediatrics, University of Navarra / University Clinic, Irunlarrea SN, Los Castaños Building, 31080 Pamplona, Spain.

E-mail: apatigar@unav.es.

\section{Objective}

To determine the influence of the genotype and the level of expression of different enzymes involved in folate metabolism on the response to and toxicity of high-dose methotrexate treatment in pediatric osteosarcomas.

\section{Study design}

DHFR and Reduced folate carrier 1 (RFC1) semiquantitative expression was analyzed in 34 primary and metastatic osteosarcoma tissues by real-time polymerase chain reaction. The following polymorphisms were also analyzed in peripheral blood from 96 children with osteosarcoma and 110 control subjects: C677T, A1298C (MTHFR), G80A (RFC1), A2756G (MTR), C1420T (SHMT), the 28bp-repeat polymorphism, and 1494del6 of the TYMS gene. Treatment toxicity was scored after each cycle according to criteria from the World Health Organization.

\section{Results}

DHFR and RFC1 expression was lower in initial osteosarcoma biopsy specimens than in metastases $(\mathrm{P}=.024$ and $\mathrm{P}=.041$, respectively). $\mathrm{RFC1}$ expression was moderately decreased in samples with poor histologic response to preoperative treatment $(\mathrm{P}=.053)$. Patients with osteosarcoma with G3/G4 hematologic toxicity were more frequently TT than CT/CC for C677T/MTHFR ( $\mathrm{P}=.023)$ and GG for A2756G/MTR (P =.048 and P $=.057$ for gastrointestinal and hematologic toxicity, respectively).

\section{Conclusions}

The role of C677T/MTHFR and A2756G/MTR on chemotherapy-induced toxicity should be further investigated in pediatric osteosarcomas receiving high-dose methotrexate. Altered expression of DHFR and RFC1 is a feasible mechanism by which osteosarcoma cells become resistant to methotrexate. 


\author{
ABBREVIATIONS \\ DHFR Dihydrofolate reductase \\ GAPDH Glyceraldehyde-3-phosphate dehydrogenase \\ mRNA \\ HD-MTX High-dose methotrexate \\ MTHFR 5,10-Methylenetetrahydrofolate reductase \\ MTR Methionine synthase \\ MTX Methotrexate \\ OR Odds ratios \\ RFC1 Reduced folate carrier I \\ SHMT Serine hydroxymethyltransferase \\ TYMS Thymidylate synthetase
}

\title{
INTRODUCTION
}

Osteosarcoma is the most common primary bone tumor in the first 3 decades of life and accounts for about $4 \%$ of all childhood tumors in Spain. ${ }^{1}$ Treatment for osteosarcoma includes surgery and preoperative and postoperative chemotherapy; for patients with nonmetastatic disease, the overall disease-free survival rate is about $65 \%$ to $70 \%{ }^{2}$ Although the disease-free survival rate has improved dramatically with the advent of chemotherapy, only $50 \%$ to $60 \%$ of tumors are chemosensitive. ${ }^{3}$

High-dose methotrexate (HD-MTX) is one of the main drugs used for osteosarcoma. MTX is a potent inhibitor of dihydrofolate reductase (DHFR), which converts dihydrofolate to the active tetrahydrofolate (Figure). In addition, the metabolized polyglutamated forms of MTX inhibit several other enzymes involved in the folate cycle, such as thymidylate synthetase (TYMS), and the combined effect is the inhibition of RNA and DNA synthesis. ${ }^{4}$

Functional polymorphisms in genes involved in the folate pathway are highly prevalent in the Caucasian population. ${ }^{5}$ In relation to cancer, the following variant genes have been analyzed in a wide variety of adult and pediatric tumors: the C677T and A1298C polymorphisms of the 5,10-methylenetetrahydrofolate reductase (MTHFR) gene, which are associated with reduced enzyme activity in vitro ${ }^{6}$; a 28 bp tandem-repeat with a $\mathrm{G}>\mathrm{C}$ substitution at the twelfth nucleotide in the second repeat of the $3 \mathrm{R}$ allele and 1494del6 polymorphisms in the TYMS gene ${ }^{7,8}$; the A2756G missense mutation (D919G) in the methionine synthase (MTR) gene ${ }^{9}$; and the C1420T polymorphism in the hydroxymethyltransferase (SHMT) gene. ${ }^{10}$

The hypothesis is emerging that somatic differences in the genes encoding the enzymes involved in folate metabolism might not only influence toxicity but also response to folate-based chemotherapy. The scarce data available indicate that the intrinsic resistance of pediatric osteosarcoma to MTX may be mediated by an impaired transport of the drug because of decreased expression of reduced folate carrier (RFC1) or the increased expression of DHFR detected in tumor samples, and that factors regulating the cell cycle, such as RB1 and E2F, may influence this effect. ${ }^{11-13}$ In addition, reduced 
levels of RFC1 in these tumors can be attributed to either decreased gene expression or presence of gene polymorphisms that could affect the protein function. An example of the latter is G80A, which could affect plasma folate and MTX concentrations. ${ }^{14}$

We hypothesized that, first, differences in the expression of folate enzymes (DHFR and RFC) in osteosarcoma tissues might be predictors of the response to MTX chemotherapy and thus, potentially, of survival; and second, that germline variations in folate pathway enzymes such as RFC, MTHFR, TYMS, SHMT, and MTR might affect the response to MTX chemotherapy or its toxicity. To test these hypotheses, we determined the genotype in 96 cases of pediatric osteosarcoma treated with HD-MTX and analyzed the level of expression of different enzymes involved in folate metabolism (Figure) in a subset $(n=34)$ of these patients for whom tumor tissues were available.

\section{METHODS}

\section{Patients and Samples}

We analyzed the genomic DNA from peripheral blood of 96 children and adolescents with osteosarcoma (Table I). For a subset of these 96, we had frozen tumor tissue: 14 biopsy specimens (from 14 patients) taken before chemotherapy and 20 metastases (from 17 patients). We analyzed the DNA from these frozen samples and from 15 samples of normal bone. For comparative purposes, 110 blood samples were obtained from adults without previous history of bone tumor who attended the Department of Orthopaedic Surgery of our center. Control subjects were approximately matched with patients in terms of the numbers of males and females but were all taken from adults to rule out development of bone tumors in late infancy/early adulthood. None of the cases or control subjects had clinical indications of congenital folate metabolism syndromes.

All the patients were treated and followed up at the Oncology Unit of the Department of Pediatrics of the University Clinic in Pamplona, Spain. In general, patients were treated before surgery with intravenous Adriamycin (doxorubicin, 3 courses at 25 to 30 $\mathrm{mg} / \mathrm{m}^{2} / \mathrm{d}$ for 3 days), intravenous methotrexate ( 4 courses of up to $14 \mathrm{~g} / \mathrm{m}^{2} / \mathrm{d}$ for 1 day), and intraarterial cisplatin ( 3 courses at $35 \mathrm{mg} / \mathrm{m}^{2} / \mathrm{d}$ for 3 days) and, after surgery, the adjuvant chemotherapy included methotrexate $\left(10 \mathrm{~g} / \mathrm{m}^{2} / \mathrm{d}\right.$ for 1 day) and alternate cycles of intravenous cisplatin/Adriamycin or intravenous actinomycin $D\left(0.45 \mathrm{mg} / \mathrm{m}^{2} / \mathrm{d}\right.$ for 3 days), cyclophosfamide (500 $\mathrm{mg} / \mathrm{m}^{2} / \mathrm{d}$ for 3 days), and vincristine $\left(1.5 \mathrm{mg} / \mathrm{m}^{2} / \mathrm{d}\right.$ for 1 day) for up to 48 weeks of treatment. Approval was granted by the Ethics Committee of the University Clinic. In all cases, informed consent was obtained from the patient, parents, or both. Clinical data were collated and made anonymous within an unlinked SPSS 15.0 database (SPSS, Chicago, Illinois). Hematologic, gastrointestinal, and renal toxicities were scored according to the World Health Organization toxicity criteria. ${ }^{15}$

\section{Semiquantitative Real-time Polymerase Chain Reaction}

RNA was isolated from tissue samples by means of the TRIzol reagents (Gibco-BRL, Invitrogen, Invitrogen S.A., Barcelona, Spain). Random primers and Superscript polymerase and reactants (Invitrogen; Invitrogen S.A., Barcelona, Spain) were used to 
reverse-transcribe RNA ( $1 \mu \mathrm{g})$ to cDNA. Semiquantitative analysis of DHFR and RFC1 mRNA expression in the tissue samples was carried out by real-time polymerase chain reaction (RT-PCR) with the ABI PRISM 7300 Sequence Detector and the software Sequence Detector version 1.4 (Perkin-Elmer/Applied Biosystems, Foster City, California).

Semiquantitative DHFR and RFC1 mRNA levels were expressed as a percentage relative to that of glyceraldehyde-3-phosphate dehydrogenase mRNA (GAPDH), which was determined with TaqMan assays (Applied Biosystems). Relative levels of expression were determined by the $\mathrm{Ct}$ method, and every assay was performed in triplicate. To determine the cut-off point for altered RFC1 and DHFR expression in osteosarcoma samples, we assayed the expression in 15 normal bone samples obtained from the same patients at tumor resection. We considered expression to be normal if it was within $3 \mathrm{SD}$ above or below the mean value of expression obtained for normal bone tissues.

\section{Genotyping of Folate Pathway Genes}

Peripheral blood samples from 96 children and adolescents with osteosarcoma and from 110 healthy adults were different. Polymorphic variants C677T and A1298C (MTHFR), G80A (RFC1), and A2756G (MTR) were detected by real-time PCR using the genotyping assays of Applied Biosystems (Applied Biosystems). SHMT1 C1420T genotypes were identified by direct sequencing of PCR products. The $28 \mathrm{bp}$ tandemrepeat and 1494del6 polymorphisms in the TYMS gene were evaluated on highresolution polyacrylamide gels after PCR.

\section{Statistical Analysis}

The $\chi^{2}$ distribution was used to compare genotype frequencies between groups (cases/control subjects, toxicity groups, and responders/non-responders). Given the lack of consensus over the most appropriate strategy for correcting for multiple tests, ${ }^{16}$ we used uncorrected $\mathrm{P}$ values. These ideally need adjustment to compensate for multiple testing, but the Bonferroni correction tended to overcompensate be-cause the markers were not all independent.

Calculation of odds ratios (OR) with a 95\% confidence interval and calculations to evaluate Hardy-Weinberg equilibrium were done with the SHEsis platform. ${ }^{17}$ In the case-control study of osteosarcomas and healthy control subjects, the power of detection of a risk of 2.25 with a significance of 5\% (2-tailed test), assuming the frequency of the polymorphic alleles present in our normal sample, ranged from $76.2 \%$ for TYMS2R/3R and RFC1-G80A, 71.3\% for MTHFR-C677T and SHMT-C1420T, 65.5\% for TYMS-1494del6 to $45.6 \%$ for MTHFR-A1298C; except for the A2756G marker of the MTR gene, for which a limited power of $15.3 \%$ was calculated.

The distributions of DHFR and RFC1 expression were nonparametric, and therefore we used the Kruskal-Wallis test to make comparisons. Overall survival data and event-free survival data were compared by means of the log rank test (Mantel-Cox test). 


\section{RESULTS}

\section{Genotype Analysis}

The genotype frequencies of the polymorphisms analyzed (MTHFR: C677T and A1298C; TYMS: 1494del6 and 5'-UTR 28 bp polymorphisms; RFC1: G80A; MTR: A2756G; and SHMT: C1420T) were in Hardy-Weinberg equilibrium (data not shown). There were no substantial case-control differences in the distribution of genotypes for the folate pathway polymorphisms (Table II).

\section{Genotype, Toxicity, and Survival}

In our series of pediatric and adolescent osteosarcomas, none of the polymorphic variants of the folate pathway enzymes was significantly related to prognosis (survival, event-free survival, development of metastasis) or to the response to treatment (tumor necrosis induced by the neo-adjuvant chemotherapy).

The toxicity induced by the antitumoral treatment was determined after every chemotherapy cycle and classified according to the guidelines of the World Health Organization. Of our patients, $70.2 \%$ suffered some kind of hematologic toxicity (aplasia, neutropenia, anemia, leukopenia) to a severe degree: grades G3/G4. In addition, $29.3 \%$ of the patients had gastrointestinal toxicity (increased alkaline phosphatase, bilirubin, or transaminases) and $42.6 \%$ had renal toxicity (creatinine, hematuria, proteinuria). Globally, neither the gastrointestinal $(\mathrm{P}=.436)$ nor the renal $(\mathrm{P}$ $=.876)$ toxicities adversely affected survival rates. There was, however, a clear negative relationship between a severe grade of hematologic toxicity and survival $(\mathrm{P}=.024, \log$ rank test).

As other researchers have detected in various solid tumors, we found some evidence of a correlation between the MTHFR C677T allele and hematologic toxicity. The statistical significance of this relationship was borderline: patients with osteosarcoma in whom severe hematologic toxicity (G3 and G4) developed were more frequently TT than $\mathrm{CT}$ or $\mathrm{CC}$ (for TT vs CT vs CC; uncorrected $\mathrm{P}=.05$; for TT vs $\mathrm{CT}+\mathrm{CC}$, OR [IC $95 \%$ ], 3.95 [1.14; 13.7], uncorrected $\mathrm{P}=.023)$.

Additionally, the GG genotype of the A2756G polymorphism (MTR) was overrepresented in the group of patients who had severe gastrointestinal toxicity (G3 and $\mathrm{G} 4$, uncorrected $\mathrm{P}=.048$ ) and also tended to be more frequent in the group of patients with hematologic toxicity (uncorrected $\mathrm{P}=.057$ ). We found no significant relationships between A1298C (MTHFR), SHMT, RFC1 or TYMS polymorphisms and hematologic, gastric, or renal toxicities.

\section{Expression Analysis}

We extracted mRNA from 15 normal bone tissue samples and analyzed the expression of DHFR and RFC1 by real-time PCR. As a reference, we used GAPDH, which was the constitutive gene used for normalization of gene expression. In this way we established the range of normal expression as the interval between $3 \mathrm{SD}$ above and below the mean 
value of expression found in normal bone tissue. The expression of DHFR and RFC1 was then analyzed in 34 samples (14 diagnostic biopsy specimens and 20 metastases) from children with bone tumors. Of the 14 biopsy samples, 13 had reduced RFC1 levels at the time of diagnosis (Table III). In terms of relative expression, the median level of expression was significantly lower in initial biopsy specimens than in metastatic tissues both for DHFR ( $\mathrm{P}=.041$, Kruskal-Wallis test $)$ and RFC1 $(\mathrm{P}=.024$, Kruskal-Wallis test).

Of the samples with a poor histologic response to preoperative treatment, $90 \%$ had decreased RFC1 expression levels. In contrast, $60 \%$ of the samples with a good histologic response had reduced RFC1 levels $\left(\mathrm{P}=.053, \chi^{2}\right.$ test $)$. We detected no evidence of a relationship between DHFR expression and response to treatment $\left(\mathrm{P}=.234, \chi^{2}\right.$ test $)$.

\section{DISCUSSION}

We identified the polymorphisms in the TYMS, MTHFR, RFC1, MTR, and SHMT genes in 96 children with osteosarcoma and 110 healthy control subjects and measured the expression of RFC1 and DHFR in 34 osteosarcoma tissue samples and 15 normal bones. Our goal was to determine how genetic variation in the folate pathway affects MTX toxicity and response in patients with osteosarcoma.

There is minimal definite information regarding the putative association between polymorphisms in the TYMS, MTHFR, RFC1, MTR, and SHMT genes and treatment outcome or MTX toxicity in osteosarcoma. With regard to an association between genotype and toxicity induced by the antitumoral treatment in our series of pediatric osteosarcomas, we detected a moderate but statistically significant relationship between the MTHFR 677TT genotype and severe degree (grades G3 and G4) of hematologic toxicity (TT vs $\mathrm{CT}+\mathrm{CC}$, uncorrected $\mathrm{P}=.023$ ). This finding is not unexpected; the same relationship has been found with other solid tumors, ${ }^{18}$ and according to a recent review article, most publications report that patients with lower MTHFR activity (MTHFR $677 \mathrm{TT}$ individuals) are subject to greater MTX-associated toxicity. ${ }^{4}$

In addition, we found that the GG genotype of the A2756G polymorphism (MTR) was moderately overrepresented in the group of patients with severe gastrointestinal toxicity (uncorrected $\mathrm{P}=.048$ ). The same genotype also tended to be more frequent in the group of patients with hematologic toxicity (uncorrected $\mathrm{P}=.057$ ). The association of this polymorphism with MTX-induced toxicity has hardly been investigated, although there are a few reports concerning patients with rheumatoid arthritis treated with low-dose MTX. In patients with rheumatoid arthritis, some authors detected an increased incidence of MTX-induced accelerated nodulosis associated with the 2756GG genotype, ${ }^{19}$ others did not detect an increased frequency of adverse effects associated with the A2756G genotype. ${ }^{20}$

In view of the number and range of discrete toxic effects observed in our patients with osteosarcoma, it would seem unlikely that the toxicity induced by the osteosarcoma treatment administered can be attributed to a single drug, such as MTX, even if administered at very high doses. Neither should one expect to explain everything with a single nucleotide polymorphism. Rather, we should look to attribute the varied toxicity 
to polymorphism in general in the metabolism, transport, and detoxification of MTX, cisplatin, and Adriamycin.

MTX resistance can occur by one or more complex mechanisms involving impaired transport into the target cell by RFC1; increase of DHFR, as a result of increased transcription or DNA amplification; and decreased intracellular retention caused by decreased polyglutamylation. ${ }^{21}$ In osteosarcoma, where high doses of MTX are required to achieve a good histologic response, all of the above mechanisms could be involved in the intrinsic resistance to MTX.

In this series of pediatric osteosarcoma, the vast majority of cases showed decreased RFC1 expression. Impaired transport of MTX by RFC1 into cancer cells therefore is the most feasible mechanism by which osteosarcoma cells become resistant to low-dose MTX. This mechanism has been proposed previously by Guo et al, ${ }^{13}$ who, as we have reported for our patients, found that a poor response to preoperative chemotherapy was significantly related to decreased expression of RFC1. Similarly, our results support those of Ifergan et al, ${ }^{22}$ who proposed that the determination of RFC1 levels in samples at the time of diagnosis might be a useful predictor of patient chemosensitivity. As already mentioned, however, MTX resistance can occur as a result of several mechanisms. Furthermore, the observed histologic response is not due to MTX alone but rather to the combined effect of MTX, Adriamycin, and intraarterial cisplatin; various studies have demonstrated the role of genetic variability in the response to the latter 2 drugs in patients with osteosarcoma. ${ }^{23,24}$

The increase of DHFR levels in metastatic tissues com-pared with primary tumors is in good agreement with the findings of other authors, ${ }^{13,21}$ who have postulated that this may be a mechanism of acquired MTX resistance or a difference between primary and metastatic tumors. In 5 resection specimens that we studied (data not shown), RFC1 and DHFR expression was more similar to that of metastatic tissues than to that of biopsy specimens. Although the small sample size cannot justify definitive conclusions, these observations support the hypothesis that an increase in RFC1 and DHFR expression reflects an acquired mechanism of the tumor cell, partially as a result of treatment rather than being intrinsic to the disease stage.

Unfortunately, we did not evaluate folate and homocysteine levels, which are an important part of the phenotypic effect of the polymorphisms examined. It would also be interesting to have specific data concerning MTX kinetics in our patients with osteosarcoma. On the other hand, one of the strengths of the study is that, despite the relatively small number of patients, there is extensive and complete clinical and toxicity data from diagnosis to disease outcome.

In conclusion, in this series of pediatric osteosarcomas receiving HD-MTX, the polymorphisms MTHFR/C677T and MTR/A2756G were associated with increased chemotherapy-induced toxicity. The reduced levels of RFC1 expression detected in osteosarcoma biopsies suggests that a feasible mechanism by which osteosarcoma cells become resistant to low-dose MTX involves impaired transport of MTX by RFC1 into the cancer cell. Further studies with series of patients with osteosarcoma for whom the folate and homocysteine levels are determined or for whom there is adequate assessment of diet and dietary supplements will be critical for drawing definite conclusions. 


\section{ACKNOWLEDGMENTS}

We are indebted to David Burdon for reviewing the English of this manuscript.

\section{REFERENCES}

1. RNTI. Registro Nacional de Tumores Infantiles. Instituto de Estudios Documentales e Históricos sobre la Ciencia (CSIC-Universitat de Valencia).

2. Link MP, Eilber F. Osteosarcoma. In: Pizzo PA, Poplack DG, editors. Principles and Practice of Pediatric Oncology. New York: Lippincott-Raven; 1997. p. 889976.

3. Lewis IJ, Nooij MA, Whelan J, Sydes MR, Grimer R, Hogendoorn PC, et al. Improvement in histologic response but not survival in osteosarcoma patients treated with intensified chemotherapy: a randomized phase III trial of the European Osteosarcoma Intergroup. J Natl Cancer Inst 2007;99:112-28.

4. Robien K, Boynton A, Ulrich CM. Pharmacogenetics of folate-related drug targets in cancer treatment. Pharmacogenomics 2005;6:673-89.

5. Sharp L, Little J. Polymorphisms in genes involved in folate metabolism and colorectal neoplasia: A HuGE Review. Am J Epidemiol 2004;159:423-43.

6. Weisberg I, Tran P, Christensen B, Sibani S, Rozen R. A second genetic polymorphism in methylenetetrahydrofolate reductase (MTHFR) associated with decreased enzyme activity. Mol Genet Metab 1998;64:169-72.

7. Kawakami K, Salonga D, Park JM, Danenberg KD, Uetake H, Brabender J, et al. Different lengths of a polymorphic repeat sequence in the thymidylate synthase gene affect translational efficiency but not its gene expression. Clin Cancer Res 2001; 7:4096-101.

8. Mandola MV, Stoehlmacher J, Zhang W, Groshen S, Yu MC, Iqbal S, et al. A 6 bp polymorphism in the thymidylate synthase gene causes message instability and is associated with decreased intratumoral TS mRNA levels. Pharmacogenetics 2004; 14:319-27.

9. Dervieux T, Greenstein N, Kremer J. Pharmacogenomic and metabolic biomarkers in the folate pathway and their association with methotrexate effects during dosage escalation in rheumatoid arthritis. Arthritis Rheum 2006;54:3095103.

10. Skibola CF, Smith MT, Hubbard A, Shane B, Roberts AC, Law GR, et al. Polymorphisms in the thymidylate synthase and serine hydroxymethyltransferase genes and risk of adult acute lymphocytic leukemia. Blood 2002;99:3786-91.

11. Serra M, Reverter-Branchat G, Maurici D, Benini S, Shen JN, Chano T, et al. Analysis of dihydrofolate reductase and reduced folate carrier gene status in relation to methotrexate resistance in osteosarcoma cells. Ann Oncol 2004;15:151-60.

12. Sowers R, Toguchida J, Qin J, Meyers PA, Healey JH, Huvos A, et al. mRNA expression levels of E2F transcription factors correlate with dihydrofolate reductase, reduced folate carrier, and thymidylate synthase mRNA expression in osteosarcoma. Mol Cancer Ther 2003;2:535-41. 
13. Guo W, Healey JH, Meyers PA, Ladanyi M, Huvos AG, Bertino JR, et al. Mechanisms of methotrexate resistance in osteosarcoma. Clin Cancer Res 1999;5:621-7.

14. Drozdzik M, Rudas T, Pawlik A, Gornik W, Kurzawski M, Herczynska M. Reduced folate carrier-1 $80 \mathrm{G}>\mathrm{A}$ polymorphism affects methotrexate treatment outcome in rheumatoid arthritis. Pharmacogenomics J 2007; 7:404-7.

15. World Health Organization. Handbook for reporting results of cancer treatment. Geneva, Switzerland: World Health Organization: Geneva, Switzerland, 1979.

16. Barroso I, Luan J, Middelberg RP, Harding AH, Franks PW, Jakes RW, et al. Candidate gene association study in Type 2 Diabetes indicates a role for genes involved in b-cell function as well as insulin action. Plos Biology 2003;1:41-5.

17. Shi YY, He L. Shesis, a powerful software platform for analyses of linkage disequilibrium, haplotype construction, and genetic association at polymorphism loci. Cell Res 2005; 15:97-8.

18. Toffoli G, Russo A, Innocenti F, Corona G, Tumolo S, Sartor F, et al. Effect of methylenetetrahydrofolate reductase $677 \mathrm{C} \rightarrow \mathrm{T}$ polymorphism on toxicity and homo-cysteine plasma level after chronic methotrexate treatment of ovarian cancer patients. Int J Cancer 2003;103:294-9.

19. Berkun Y, Abou Atta I, Rubinow A, Orbach H, Levartovsky D, Aamar S, et al. 2756GG genotype of methionine synthase reductase gene is more prevalent in rheumatoid arthritis patients treated with methotrexate and is associated with methotrexateinduced nodulosis. J Rheumatol 2007;34:1664-9.

20. Wessels JA, Kooloos WM, De Jonge R, De Vries-Bouwstra JK, Allaart CF, Linssen A, et al. Relationship between genetic variants in the adenosine pathway and outcome of methotrexate treatment in patients with recent-onset rheumatoid arthritis. Arthritis Rheum 2006;54:2830-9.

21. Assaraf YG. Molecular basis of antifolate resistance. Cancer Metastasis Rev 2007;26:153-81.

22. Ifergan I, Meller I, Issakov J, Assaraf YG. Reduced folate carrier protein expression in osteosarcoma: implications for the prediction of tumor chemosensitivity. Cancer 2003;98:1958-66.

23. Mintz MB, Sowers R, Brown KM, Hilmer SC, Mazza B, Huvos AG, et al. An expression signature classifies chemotherapy-resistant pediatric osteosarcoma. Cancer Res 2005;65:1748-54.

24. Ochi K, Daigo Y, Katagiri T, Nagayama S, Tsunoda T, Myoui A, et al. Prediction of response to neoadjuvant chemotherapy for osteosarcoma by geneexpression profiles. Int J Oncol 2004;24:647-55. 


\begin{tabular}{|c|c|}
\hline \multicolumn{2}{|c|}{$\begin{array}{l}\text { Table 1. Clinicopathologic features of } 96 \text { patients } \\
\text { with osteosarcoma }\end{array}$} \\
\hline & Mean (SD) or Frequency (\%) \\
\hline \multicolumn{2}{|l|}{ Sex } \\
\hline Male & $41(42.7 \%)$ \\
\hline Female & $55(57.3 \%)$ \\
\hline \multicolumn{2}{|l|}{ Histologic study } \\
\hline Osteoblastic & $52(54.2 \%)$ \\
\hline Chondroblastic & $16(16.7 \%)$ \\
\hline Other & $28(29.2 \%)$ \\
\hline \multicolumn{2}{|l|}{ Location } \\
\hline Extremity & $91(94.8 \%)$ \\
\hline Central & $5(5.2 \%)$ \\
\hline \multicolumn{2}{|l|}{ Metastasis } \\
\hline No & $49(51.3 \%)$ \\
\hline At diagnosis & $18(18.6 \%)$ \\
\hline At follow-up & $29(30.1 \%)$ \\
\hline \multicolumn{2}{|l|}{ Response to treatment } \\
\hline Good ( $\geq 90 \%)$ & $48(50 \%)$ \\
\hline Poor $(<90 \%)$ & $39(40.6 \%)$ \\
\hline Not available & $9(9.4 \%)$ \\
\hline Age at diagnosis (years) & $14.5(4.74)$ \\
\hline Overall survival* & $112.8(4 ; 262)$ \\
\hline Event-free survival $\uparrow$ & $102.7(0 ; 262)$ \\
\hline \multicolumn{2}{|c|}{$\begin{array}{l}\text { *Overall survival: time in months from diagnosis until death or } \\
\text { until the study’s end-date (ie, length of follow-up) expressed as } \\
\text { median (range). } \\
\dagger \text { Event-free survival: time in months from diagnosis until } \\
\text { clinical remission or first event (death, metastasis or relapse) } \\
\text { expressed as median (range). }\end{array}$} \\
\hline
\end{tabular}


Table 2. Genotype frequencies, odds ratios, and associated probabilities for polymorphisms in TYMS, MTFHR, SHMT, MTR, and RFC1 genes in the blood samples of children with osteosarcoma and in control subjects

\begin{tabular}{|c|c|c|c|c|}
\hline & $\begin{array}{c}\text { Control subjects } \\
(\mathrm{n}=110)\end{array}$ & $\begin{array}{c}\text { Osteosarcomas } \\
(\mathbf{n}=96)\end{array}$ & OR (CI) & $P$ value \\
\hline \multicolumn{5}{|l|}{ TYMS 3R-2R } \\
\hline $3 \mathrm{R} / 3 \mathrm{R}$ & $26(23.6 \%)$ & $30(31.3 \%)$ & $1.26(0.57 ; 2.8)$ & .56 \\
\hline $3 \mathrm{R} / 2 \mathrm{R}$ & $62(56.4 \%)$ & $46(47.9 \%)$ & $0.83(0.41 ; 1.7)$ & .609 \\
\hline $2 \mathrm{R} / 2 \mathrm{R}$ & $22(20 \%)$ & $20(20.8 \%)$ & 1 & Referent* \\
\hline $3 R / 3 R$ vs $3 R 72 R+2 R / 2 R$ & & & $1.45(0.8 ; 2.6)$ & .084 \\
\hline \multicolumn{5}{|l|}{ TYMS, 1494del6 } \\
\hline ins/ins & $49(44.5 \%)$ & $46(47.9 \%)$ & 1 & Referent* \\
\hline ins/del & $44(40 \%)$ & $35(36.5 \%)$ & $0.84(0.45 ; 1.5)$ & .576 \\
\hline del/del & $17(15.5 \%)$ & $15(15.6 \%)$ & $0.93(0.41 ; 2.1)$ & .873 \\
\hline \multicolumn{5}{|l|}{ MTHFR, C677T } \\
\hline $\mathrm{CC}$ & $35(31.8 \%)$ & $35(36.5 \%)$ & 1 & Referent* \\
\hline $\mathrm{CT}$ & $54(49.1 \%)$ & $47(49 \%)$ & $0.87(0.47 ; 1.6)$ & .656 \\
\hline TT & $21(19.1 \%)$ & $14(14.6 \%)$ & $0.67(0.3 ; 1.5)$ & .333 \\
\hline \multicolumn{5}{|l|}{ MTHFR, A1298C } \\
\hline AA & $66(60 \%)$ & $53(55.2 \%)$ & 1 & Referent* \\
\hline $\mathrm{AC}$ & $35(31.8 \%)$ & $36(37.5 \%)$ & $1.26(0.7 ; 2.3)$ & .44 \\
\hline $\mathrm{CC}$ & $9(8.2 \%)$ & $7(7.3 \%)$ & $1.1(0.36 ; 3.1)$ & .898 \\
\hline \multicolumn{5}{|l|}{ SHMT, C1420T } \\
\hline $\mathrm{CC}$ & $49(44.5 \%)$ & $35(36.4 \%)$ & 1 & Referent* \\
\hline $\mathrm{CT}$ & $39(35.4 \%)$ & $40(41.7 \%)$ & $1.48(0.8 ; 2.7)$ & .21 \\
\hline TT & $21(19.1 \%)$ & $21(21.9 \%)$ & $1.48(0.7 ; 3.1)$ & .305 \\
\hline \multicolumn{5}{|l|}{ RFC1, G80A } \\
\hline $\mathrm{GG}$ & $29(26.4 \%)$ & $29(30.2 \%)$ & 1 & Referent* \\
\hline GA & $55(50 \%)$ & $49(51 \%)$ & $0.9(0.47 ; 1.7)$ & .739 \\
\hline $\mathrm{AA}$ & $26(23.6 \%)$ & $18(18.8 \%)$ & $0.7(0.32 ; 1.52)$ & .453 \\
\hline \multicolumn{5}{|l|}{ MTR, A2756G } \\
\hline AA & $78(70.9 \%)$ & $72(75 \%)$ & 1 & Referent* \\
\hline $\mathrm{AG}$ & $30(27.3 \%)$ & $23(24 \%)$ & $0.83(0.44 ; 1.55)$ & .556 \\
\hline GG & $2(1.8 \%)$ & $1(1 \%)$ & $0.56(0.06 ; 5.45)$ & .591 \\
\hline
\end{tabular}

CI, Confidence interval.

*Genotype considered to be the reference group, with an $\mathrm{OR}=1$. 
Table 3. Level of expression of DHFR and RFC1 in primary osteosarcoma tissues and metastases

\begin{tabular}{|c|c|c|c|c|c|c|}
\hline \multirow{2}{*}{ Tissue type } & \multirow{2}{*}{ No. } & \multicolumn{3}{|c|}{ DHFR $(P=.062)$} & \multicolumn{2}{c|}{ RFC1 (P=.1) } \\
\cline { 3 - 7 } & & Low* & Normal* & High* & Low* & Normal* \\
\hline Biopsy & 14 & $9(64.3 \%)$ & $4(28.6 \%)$ & $1(7.1 \%)$ & $13(92.9 \%)$ & $1(7.1 \%)$ \\
\hline Metastases & 20 & $6(30 \%)$ & $6(30 \%)$ & $8(40 \%)$ & $11(68.8 \%)$ & $5(31.3 \%)$ \\
\hline
\end{tabular}

*The ratio of expression of DHFR or RFC1 relative to GAPDH in the tumor tissue was within the interval of $3 \mathrm{SD}$ above and below the mean of the normalized expression in normal bone tissue. Low: below the normal range. High: above the normal range. 


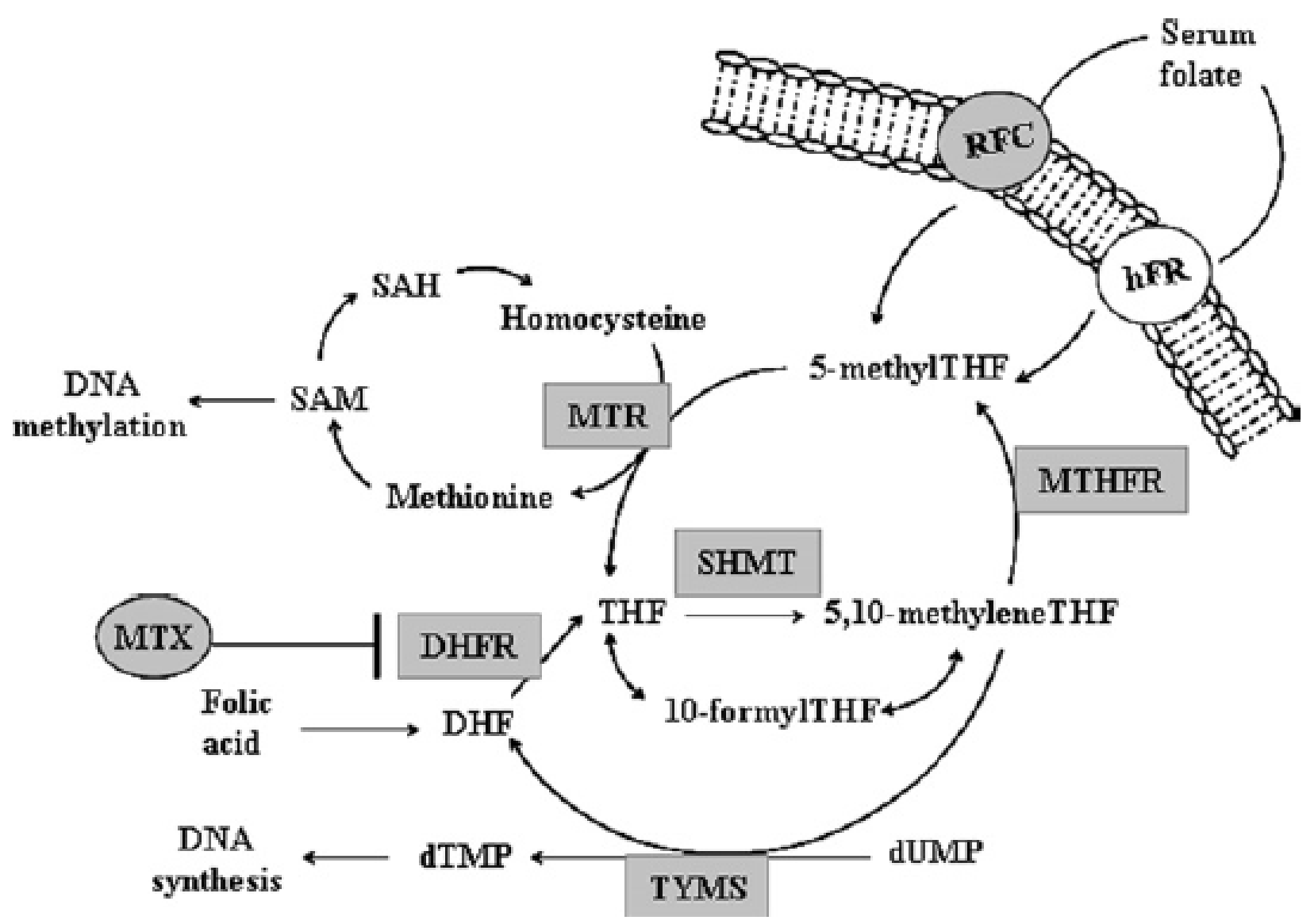

Figure. Overview of folate metabolism and transport. The enzymes analyzed in this study are highlighted. 